\title{
FGF2: a key regulator augmenting tendon-to-bone healing and cartilage repair
}

\author{
Jun Zhang1 (iD), Ziming Liu², Yuwan Li ${ }^{3}$, Qi You', Jibin Yang', Ying Jin', Gang Zou', \\ Jingfeng Tang ${ }^{1}$, Zhen Ge ${ }^{1}$ \& Yi Liu*,1 \\ ${ }^{1}$ Department of Orthopaedic Surgery, Affiliated Hospital of Zunyi Medical University, Guizhou 563000, China \\ ${ }^{2}$ Peking University Institute of Sports Medicine, Beijing 100083, China \\ ${ }^{3}$ Department of Orthopaedic Surgery, The First Affiliated Hospital of Chongqing Medical University, Chongqing 400016, China \\ *Author for correspondence: 13308529536@163.com
}

Ligament/tendon and cartilage injuries are clinically common diseases that perplex most clinicians. Because of the lack of blood vessels and nerves, their self-repairing abilities are rather poor. Therefore, surgeries are necessary and also widely used to treat ligament/tendon or cartilage injuries. However, after surgery, there are still many problems that affect healing. In recent years, it has been found that exogenous FGF2 plays an important role in the repair of ligament/tendon and cartilage injuries and exerts a synergistic effect with endogenous FGF2. Therefore, FGF2 can be used as a new type of biomolecule to accelerate tendon-to-bone healing and cartilage repair after injury.

First draft submitted: 27 June 2019; Accepted for publication: 19 October 2020; Published online: 17 November 2020

Keywords: cartilage $\bullet$ cartilage injury $\bullet$ cartilage repair $\bullet$ FGF $\bullet$ FGF2 $\bullet$ FGFR $\bullet$ ligament $\bullet$ tendon $\bullet$ tendon-to-bone healing • tissue engineering

Tendon/ligament injuries, caused by trauma, physical sports or routine daily activities, are prevalent conditions in orthopedic clinical practices with a high morbidity worldwide [1]. Among these, anterior cruciate ligament (ACL) and rotator cuff injuries are the most common [1]. In the United States, the incidence of ACL injury is estimated to be approximately 100,000-200,000, which means that around 1 in 3000 Americans suffers from an ACL injury $[2,3]$. Although the exact incidence of rotator cuff injuries is still unclear, it may be approximately 5$30 \%$, and full-thickness tears have been observed to be as high as $30 \%$ based on cadaver research using elderly volunteers $[4,5]$.

Depending on a patient's occupation, severity of injury and assistance needs or functional state, different therapies are used to treat ACL injuries and rotator cuff tears. Although surgical reconstruction has been widely used to treat ACL ruptures and severe rotator cuff tears, effective tendon-to-bone healing after surgery directly determines the success of the operation and future function. After graft implantation, inflammatory cells, including neutrophils and macrophages, are present in the tendon-to-bone interface. Later, cytokines generated by the inflammatory cells play important roles in the formation of fibrous scar tissue between the graft and host bone [6].

The native ligamentous/tendinous insertion to the bone is an extremely specialized and highly organized hierarchical structure that facilitates the transmission of intricate mechanical force from ligament/tendon to bone. The representative structure of tendon-to-bone interface consists of four explicit zones: dense connective tissue (tendon/ligament), uncalcified fibrocartilage, calcified fibrocartilage and bone (Figure 1) [7-11]. The tendon-to-bone interface heals more slowly and incompletely compared with bone-to-bone healing because of the comparative avascularity of the fibrocartilage zone and bone loss at the site of injury [12]. Although reconstructive surgeries to repair tendon-to-bone injuries have been mastered by clinicians and are performed extensively, graft failure continues to occur, which may relate to non-traumatic causes or traumatic injuries. Non-traumatic causes include poor healing of the tendon-to-bone interface, fixation failure and technical errors [6]. For instance, the failure rate of knee posterolateral repair is $37-40 \%$, and that of rotator cuff tear repair is $20-90 \%$ [13]. Therefore, improvement in the speed and success of tendon-to-bone healing after reconstruction and acceleration of osseointegration are current research hot spots in the field of sports medicine.

Future Medicine 


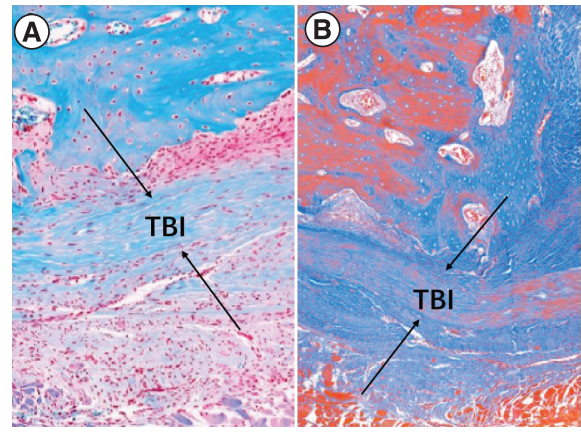

Figure 1. The representative structure of the tendon-to-bone interface consists of four explicit zones: dense connective tissue (tendon/ligament), uncalcified fibrocartilage, calcified fibrocartilage and bone. (A) Safranin O/fast green staining. (B) Masson's trichrome staining.

TBI: Tendon-to-bone interface.

Over the past decade, various treatments to biologically improve and accelerate tendon-to-bone healing have been systematically and rigorously studied in the field of orthopedic fundamental research, principally aiming to enhance biological healing of the tendon-to-bone interface using tissue engineering approaches, including mesenchymal stem cells (MSCs), growth factors and scaffolds. In addition, many other therapies, including platelet-rich plasma, gene therapy, grafts enveloped with periosteum and low-intensity pulsed ultrasound, have been used to accelerate tendon-to-bone healing [14-23]. Many researchers have developed tendon-to-bone sites that are similar to normal tissue by increasing graft activity, accelerating graft revascularization and reconstructing the biomechanical properties of the graft $[14,16,21]$.

Articular cartilage is located on the surface of connected bones in the joint. Because of its physiological characteristics, such as weight-bearing, low friction and wear resistance, articular cartilage enables painless articulation of the skeleton [24]. Thus, articular cartilage plays an important role in protecting joints from injury. Articular cartilage, which is primarily composed of chondrocytes, with extracellular matrix (ECM) outside it [25], lacks blood vessels, lymphatic vessels and nerve fibers. The main components of the ECM are water, type II collagen and proteoglycans [26], which are mainly produced and maintained by chondrocytes. Because of the special structure of articular cartilage, limited sources of nutrients and oxygen and the presence of a hard ECM, when cartilage tissue is damaged, it is difficult for chondrocytes in the cartilage ECM to migrate to the damaged cartilage sites. In addition, lack of vascularization contributes to the poor capacity of articular cartilage to recover from damage. Therefore, its self-repair ability is very limited [27]. At present, the main treatment methods for articular cartilage injury include drug therapy (e.g., nonsteroidal anti-inflammatory drugs, glucosamine), cell-free biomimetic scaffolds and microfracture surgery [28-34]. Autologous/allogeneic osteochondral transplantation and matrix-induced autologous chondrocyte implantation or autologous chondrocyte implantation have also been widely used as treatments for cartilage injuries [35-38]. However, conservative treatments are only appropriate for minor cartilage injuries. Although cell-free biomimetic scaffolds avoid cell extraction and expansion, the quality of subchondral bone is poor [39]. Microfracture offers effective short-term symptomatic improvement, but more fibrous regenerated tissue, along with reoccurrence of clinical symptoms, is seen as early as 2 years after operation [40]. Although autologous chondrocyte implantation has shown good outcomes in a prospective randomized trial [38], many challenges still exist, including high treatment cost, graft hypertrophy and need for two surgeries [35,36]. Osteochondral transplantation (autograft or allograft) has shown a good clinical result in the short term, but more failures ( 55 vs $17 \%$ ) occur after osteochondral transplantation compared with autologous chondrocyte implantation by 10 years post-surgery [41]. So far, there is no consensus with regard to the most effective form of treatment.

FGF2, which is also known as bFGF, is a member of the fibroblast family of factors and is widely distributed in the body, with a high affinity for heparin [42]. As a mitotic promoter, it can promote cell mitosis and accelerate cell proliferation. It has been widely used in various tissue repair studies, such as those investigating tendon-to-bone healing [43], articular cartilage damage repair [44-46] and bone injury repair [47]. This review intends to look at the application of FGF2 in tendon-to-bone healing and cartilage repair.

\section{FGF family members}

FGFs, which are also known as HBGFs, are polypeptide growth factors that are extensively expressed in developing and adult tissues. They can interact with specific receptors on cell membranes to mediate various biological and pathophysiological processes, including embryonic development, angiogenesis, mitogenesis, metabolism regulation, cellular differentiation, cell migration and tissue injury repair [48,49]. 
At present, 22 FGF-encoding genes have been identified in the human genome. The human FGF gene family can be divided into seven subfamilies according to phylogenetic analysis [49]. Therefore, the members of each subfamily share many similarities in sequence, biochemical properties and developmental characteristics. Most chromosomal locations of human FGF genes have been identified, except those of FGF16. FGF genes have an approximately $25-50 \%$ homologous amino acid sequence, which means they contain about 120 identical amino acids. Their homologous amino acid sequences fold into a spherical $\beta$-trilobal shape, which determines the similar biological effects between their different molecular structures [50]. However, their core domains are rather conserved, containing distinctly different amino-terminal and carboxy-terminal sequences, which confer unique functional properties on members of the FGF family to some extent. The secretory modes of FGFs primarily include paracrine and autocrine/endocrine. Paracrine has a regular $\beta$-trilobal domain composed of $\beta 12$ chains, whereas autocrine lacks the typical $\beta$-triple because of a lack of $\beta 11$ chains [48]. Human and bovine $F G F 1$ has 140 amino acids, and the molecular weight of $F G F 1$ is $15.5 \mathrm{kDa}$ [51]. Xenopus FGF1 has a total length of 154 amino acids, and the lysine at position 132 is the most easily chemically modified [51]. The murine FGF4 gene has at least four cis-regulatory factors that control its expression in mouse embryonal carcinoma and embryonic stem cells [52]. Human $F G F 1$ and FGF2 genes are located on chromosomes 4 and 5, respectively, both of which are single-copy genes [53].

The activities of FGFs are regulated by diverse mechanisms, including combination with heparan sulfate, $\mathrm{N}$-terminal alternative splicing, homodimerization and site-specific proteolytic cleavage of FGF ligands. The interaction between FGFs and heparan sulfate is the most crucial factor determining the biological activities of the ligands. It determines not only the way FGFs work but also the different biological activities of paracrine ligands in the same subfamily [50]. In terms of mechanism, heparan sulfate mainly plays a role in the following ways: by acting as a storage reservoir for FGF interaction with FGFRs; by assisting the FGF-FGFR interaction by restricting dimensionality of FGF ligands; and by protecting FGF from $\mathrm{pH}$-dependent, proteolytic and thermal degradation. Paracrine FGFs play biological roles by activating FGFRs and interacting with heparan sulfate domains on the cell surface. Although endocrine FGFs also depend on FGFRs to exert their functional effects, they mainly rely on $\alpha / \beta$-Klotho co-receptors [54].

\section{FGFRs}

FGFRs are typical transmembrane tyrosine kinase receptors that are expressed in most tissue cells and mediate the transmission of FGFs into the cytoplasm. Thus far, four FGFRs have been discovered [49]: FGFR1, FGFR2, FGFR3 and FGFR4. They are mainly composed of three parts: a ligand domain (D1-D3) composed of three immunoglobulin-like repeats outside the cell, a transmembrane domain composed of hydrophobic amino acids and a tyrosine protein kinase activity domain in the cell [50]. When FGF binds to an FGFR, the tyrosine protein kinase activity domain in the cell first undergoes self-phosphorylation. Subsequently, the target protein of the FGFR is trans-phosphorylated. This signal is transmitted to the nucleus through a cascade reaction $[50,55,56]$ and plays an important role in injury repair, vascular and nerve regeneration, embryo development and bone formation. FGFR1, FGFR2 and FGFR3 all express in normal and injured ligaments. After ligament injury, FGFR2 initially upregulates and then returns to normal 7 days after injury, whereas FGFR1 and FGFR3 continue to be unchanged after injury. The transient expression of and signaling through FGFR2 promote the proliferation of fibroblasts and endothelial cells and further promote the healing of injured ligaments. It is pivotal for targeted exogenous FGF treatment to make full use of FGFR2 expression 3 days after ligament injury [57]. Of the four FGFRs, human adult articular chondrocytes mainly express FGFR1 and FGFR3 [58]. In osteoarthritis patients, the expression of FGFR3 substantially decreases while the expression of FGFR1 increases, which indicates the roles of FGFR1 and FGFR3 in articular cartilage degeneration. FGFR1 has a dominant function activated by FGF2 in cartilage degeneration of osteoarthritis patients [58]. A relevant study also found that the mutation of the FGFR3 gene might result in achondroplasia [59].

\section{FGF2: discovery \& application}

FGF2 was one of the first members of the FGF family to be discovered. The human FGF2 gene is a single-copy gene located on the short arm of chromosome 4 with a total length greater than $40 \mathrm{~kb}$. Noncoding sequences regulate gene transcription at the $5^{\prime}$ and $3^{\prime}$ ends of the gene [60]. FGF2 is widely distributed in the body, including in most organs, tissues and cells. Because of its extensive distribution in the body and strong cell-promoting ability, FGF2 plays an important role in tendon-to-bone healing, cartilage repair, bone repair and nerve regeneration. The mechanism of FGF2 is shown in Figure 2. 


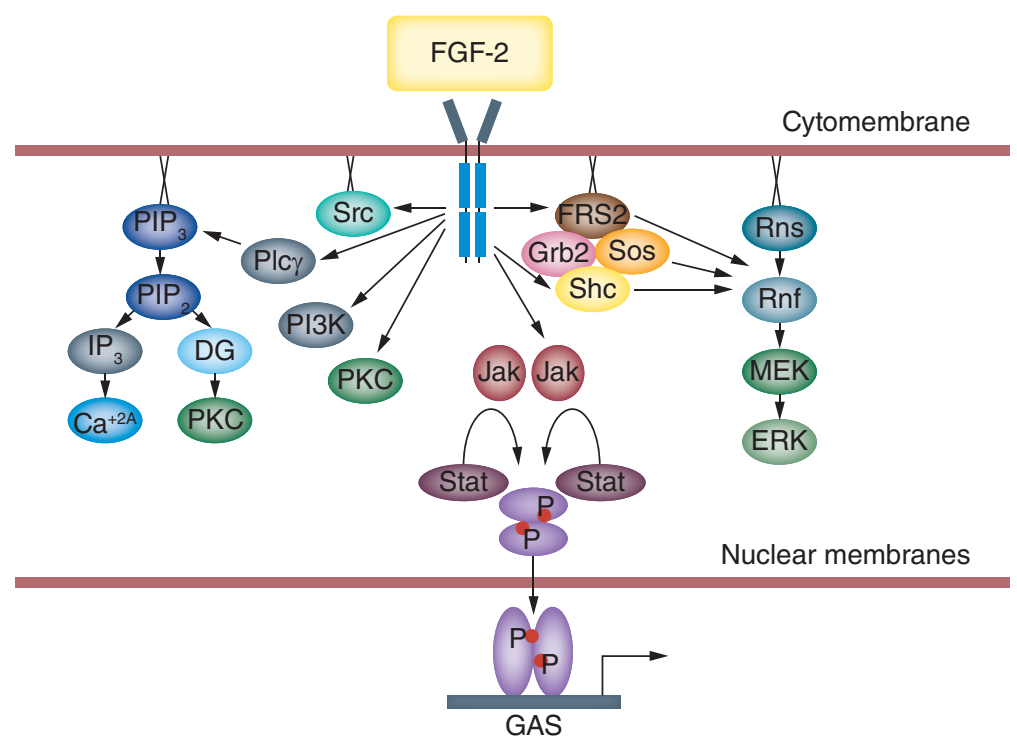

Figure 2. The mechanism of action of FGF2. FGF2 can specifically bind to tyrosine kinase receptors and activate the FGF/FGFR signaling pathway. Thereby, FGF2 initiates various pathways of this signal transduction and ultimately participates in many important biological processes, such as cell proliferation, differentiation and apoptosis and immune regulation. This includes the JAK-STAT signaling pathway, which has been proven to be closely related to cartilage metabolism [61,62].

\section{The effect of FGF2 on tendon-to-bone healing}

According to statistics, approximately 200,000 cases of ACL injury in the United States require surgical treatment [63]. Thus far, surgical reconstruction has been widely used to treat ACL rupture. The main grafts used in clinical practice include autologous ligaments, allogeneic ligaments and artificial ligaments. However, about $0.7-10 \%$ of patients require secondary surgery because of surgical failure, which may result from the slow growth and healing of the tendon-to-bone interface. In addition to the significance of anatomic reconstruction of the ligament, the morphological duplication of the characteristic tendon-to-bone insertion site may play an important role in the progress of tendon-to-bone healing.

Tendon-to-bone healing after ACL reconstruction is a complex, gradual evolutionary biological process. A graft must undergo the three stages of hematoma, necrosis and revascularization and structural remodeling to form the four-layer structure of a normal ligament end: tendon/ligament, uncalcified fibrocartilage, calcified fibrocartilage and bone tissue. In the process of tendon-to-bone healing, various cell- and cytokine-mediated inflammatory responses, cell proliferation, fibrocartilage/bone tissue formation and angiogenesis play key roles. Any factors that affect revascularization, cell proliferation and matrix remodeling of grafts may result in the failure of tendon-to-bone healing. Therefore, improvement in the speed and success of tendon-to-bone healing after ACL reconstruction and acceleration of osseointegration are current research hot spots in the field of sports medicine.

FGF2 is an important growth factor in the healing process of ligament/tendon injury [64], and the expression of FGF2 highly increases after ligament/tendon injury [64,65]. After ligament/tendon reconstruction, the expression of FGF2 peaks at 1 week [64]. In the early stage of ligament tissue healing after reconstruction of acute rotator cuff tears in rabbits, ligament cells and tendon sheath cells begin to secrete endogenous FGF2 [65], which can promote cell proliferation, stimulate cell migration and angiogenesis and increase the expression of type I/III collagen. Therefore, adding exogenous FGF2 within the first 10 days (the early stage of injury repair) after ligament injury can produce synergistic effects with endogenous FGF2 and promote cell proliferation, ECM formation and remodeling of the ECM, which can significantly shorten the time of tendon-to-bone healing [66]. Hankemeier $e t$ al. analyzed the effect of low-dose $(3 \mathrm{ng} / \mathrm{ml})$ FGF2 and high-dose FGF2 $(30 \mathrm{ng} / \mathrm{ml})$ on proliferation, differentiation, cell density and apoptosis of human bone marrow-derived MSCs (BM-MSCs) and compared the results with those of a control group without FGF2 [66]. They found that low-dose FGF2 had the capacity to stimulate both proliferation and differentiation of BM-MSCs. By contrast, high-dose FGF2 did not stimulate proliferation and gene expression of ECM proteins. These findings might be beneficial for both cell culturing and engineering of bioartificial tendons and ligaments. The researchers also found that a low dose of FGF2 $(3 \mathrm{ng} / \mathrm{ml})$ generated a biphasic effect on BM-MSCs. After 7 days, the cell proliferation rate reached its maximum and was higher compared with that seen in the other groups. Moreover, type I/III collagen and fibronectin mRNA expression was overtly upregulated with low-dose FGF2 on days 14 and 28. Interestingly, a high dose of FGF2 did not promote differentiation or proliferation of BM-MSCs. 
Zhang et al. demonstrated that sustained release of FGF2 by fibrin gel carriers could promote the differentiation of BM-MSCs into ligament in vitro and in vivo and promoted patellar ligament healing of rabbits [67]. In their study, they found that FGF2- and CaPP-loaded fibrin clots could upregulate the mRNA expression of ALP, RUNX2, VEGF, OPN and collagen I. VEGF is beneficial to the revascularization of allografts. ALP and OPN are crucial markers of osteogenic differentiation. RUNX2 is known as a key regulator of osteogenic lineage. Moreover, collagen I is an important factor in optimal tendon-to-bone healing. Further in vivo research found that FGF2-loaded fibrin clots could improve the connection between patella and patellar tendon compared with the control group [67]. FGF2 enhanced cell differentiation and the expression of bone- and ligament-related genes, which enhanced the bioactivity of the tendon-to-bone interface and resulted in the histological improvement of tendon-to-bone healing. Type I/III collagen is crucial for maintaining the mechanical strength of ligaments and tendons, and the results of studies by Hankemeier $e$ al. and Zhang et al. demonstrated that FGF2 could induce mRNA upregulation of either type I or type III collagen [66,67]. Therefore, ultimate improvement in function might be closely related to the mRNA upregulation of type I or type III collagen.

The appearance and development of tissue-engineered ACL allografts have provided a new direction and prospect for the treatment of ACL injuries. The use of decellularized ACL allografts in ACL replacement surgery contributes to recapturing the function of the ligament after surgery and accelerating tendon-to-bone healing by retaining the ligament's native structures. Previous studies have found that decellularized ACL that was repopulated with the host's own ACL fibroblasts might minimize extrinsic cell invasion and preserve the mechanical properties of the graft, but the repopulation was noted to be low [68]. MacLean et al. wanted to increase the repopulation levels of ACL fibroblasts in young pigs using different concentrations of FGF2 [68]. They found that $6 \mathrm{ng} / \mathrm{ml} \mathrm{FGF2} \mathrm{was} \mathrm{the}$ most appropriate concentration for increasing cellular growth in decellularized ACL, whereas 0.1 and $60 \mathrm{ng} / \mathrm{ml}$ FGF2 did not improve cellular repopulation of decellularized ACL. Their results also demonstrated that FGF2 could promote ACL healing and accelerate tendon-to-bone healing after ACL reconstruction.

Compared with the use of recombinant molecules, gene transfer of therapeutic elements in ligaments and tendons is a prospective strategy for continuously regulating the healing processes of injured tissues [69-72]. After ligament injuries, such gene-based methods can regulate a series of biochemical changes, such as variations in wound contractile $\alpha$-smooth muscle actin and NF- $\mathrm{kB}$ markers and expression of type I and type III collagen in rabbits [73,74]. Viral gene delivery vectors and nonviral gene delivery vectors have been used to transfer specific genes into cells. Many studies have found that recombinant adeno-associated virus (rAAV) vectors can transduce cells from various species (e.g., chicken, rat, rabbit and mouse) both in vitro and in vivo [75-78], and rAAV is the most popular system in human gene therapy methods because of its unique advantages. For example, rAAV carries no viral coding sequences, so it is less immunogenic compared with adenoviruses. In addition, rAAV remains mostly episomal compared with retroviruses, and thus insertional mutagenesis is more likely to occur. The outcomes and advantages of offering genes for PDGF [79-82], bone morphogenetic proteins (BMPs) [83-87], GDF5 [88], IGF1 [89,90], TGF- $\beta$ [91-93] and scleraxis [94] in tissue healing have been largely described in various systems in vitro, in situ and in vivo. Madry et al. focused on delivering FGF2 to human ligament cells and explants via rAAV [95]. They wanted to explore whether FGF2 gene transfer with this vector had the potential to accelerate the healing process of human ACL injuries. They found that rAAV-mediated delivery allowed for significant, durable production of FGF2 in both human ACL fibroblasts in vitro and explant cultures in situ. Moreover, the levels of FGF2 generated through rAAV gene transfer allowed for sustained and steady stimulation of cell proliferation and critical metabolic processes (such as type I/III collagen deposition and balanced collagen I:III ratios). Moreover, Wang et al. used AAV-mediated gene transfer to deliver FGF2 gene to tenocytes and found that the levels of expression of FGF2 and type I/III collagen had upregulated [77], which was in good agreement with the conclusions of Madry et al. [95].

Treatment of the disrupted intrasynovial flexor tendon is another problem that perplexes many clinicians and may be complicated by the formation of adhesions and rupture because of weak repairs. The primary cause of unsatisfactory results is limited healing ability. The transfer of target genes integral to the reparative process provides an effective approach to accelerating the healing of injured tendon. Tang et al. successfully transferred $F G F 2$ to the injured digital flexor tendons of white leghorn chickens via the AAV2 vector and explored its effect on the healing strength of the tendons as well as adhesion formation in a clinically relevant injury model [96]. Interestingly, the FGF2 gene, transferred to the digital flexor tendons via the AAV2 vector, enhanced the healing strength during pivotal tendon healing stages and did not enhance adhesion formation. Therefore, it is possible to increase the healing strength of injured tendons and prevent adhesion formation with this method. Yang et al. used poly(lacticco-glycolic acid) nanoparticles as vehicles to deliver FGF2 and VEGFA genes into injured tendon tissues [97]. They 
also found that the ultimate strength of repaired tendons treated with the nanoparticle/FGF2/VEGFA plasmid compound was significantly enhanced, indicating that combination therapy could increase flexor tendon gliding function. It should be noted that maximum strength and gliding function are the two factors that determine postoperative function of repaired tendons. Gliding function is related to adhesion formation, and less adhesion formation results in better gliding function.

The core of tendon-to-bone healing is not only the vascularization and regeneration of the reconstructed graft but also the formation and mineralization of new bone, which plays a key role in narrowing the bone tunnel and accelerating the healing process. Yin $e t$ al. used periosteum-derived stem cells as seed cells and adhered them to chitosan/collagen scaffolds to observe the repair of Sprague Dawley rat skull defects [98]. The scaffolds were capable of continuously releasing FGF2 and BMP2. The experimental results showed that the repair effect of the experimental group was significantly better than that of the control group, suggesting that FGF2 and BMP2 provide a better environment for the proliferation and differentiation of seed cells and improve the efficiency of bone repair. Although the mechanism of skull bone formation (mainly direct, intramembranous ossification) is different from that observed in subchondral bone formation (endochondral ossification), this experiment suggested that FGF2 played an important role in promoting MSC differentiation and laid an important foundation for further research of bone regeneration except form skull bone formation. Moreover, Ikpegbu et al. demonstrated that FGF2 could increase the phenotype of osteocytes and promote the differentiation of osteoblasts [99]. Osteocytes are located in bone tissue, and osteoblasts are located on the edge of bone tissue. When bone grows and regenerates, osteoblasts are arranged in a regular layer on the surface of bone tissue and secrete matrix and fibers to the surrounding bone. Osteoblasts embed themselves in the secreted matrix and fibers and form osteoid. When bone salts are deposited, osteoblasts become bone tissue, and osteoblasts mature into osteocytes and promote the transformation of osteoblasts into osteocytes, thus accelerating the process of bone damage repair, which is mainly achieved by upregulating the expression of the downstream E11 protein. Therefore, the FGF2-E11 signaling pathway may also be involved in skeletal development and other diseases, and its mechanism requires further investigation. In addition, the effects on osteocyte phenotype may well be associated with specific FGF2 receptor activation. Among FGFRs, FGFR1 is mainly expressed in osteoblasts and has the function of regulating their growth and development [100]. FGFR1, as a transmembrane protein, can bind to its ligand and introduce stimulation into cells and then regulate osteoblasts at various stages by regulating multiple signaling pathways related to bone metabolism. The MAPK cascade signaling pathway is the most important signaling pathway [101].

Lee $e t$ al. also studied the effect of different levels of FGF2 on the repair of bone defects in New Zealand white rabbits [102]. At different time points, different concentrations of FGF2 promoted the growth of new bone, suggesting that FGF2 had a promoting effect on the repair of bone defects in New Zealand white rabbits. At 2 weeks, no difference was observed in all groups. At 4 weeks, the 1 and $0.5 \mathrm{mg} / \mathrm{ml} \mathrm{FGF2} \mathrm{groups} \mathrm{showed} \mathrm{more} \mathrm{new}$ bone formation compared with the control group. At 12 weeks, the $1,0.5$ and $0.1 \mathrm{mg} / \mathrm{ml}$ groups all had more new bone formation compared with the control group [102]. The reason and possible mechanism were as follows: high concentration of FGF2 had a more positive effect on new bone formation and bone regeneration, and bone regeneration or healing was a dynamic process - there was increased new bone formation as time went by [102].

\section{The effect of FGF2 on cartilage repair}

The self-repair ability of cartilage is very limited. Therefore, it is necessary to intervene by various means after cartilage damage occurs. Although current therapies have achieved some results, it is also necessary to explore new treatments to maximize patient satisfaction and long-term outcomes. Many growth factors have been studied with regard to cartilage repair. Growth factors are related to cartilage development, proliferation of chondrocytes and BM-MSCs, chondrogenic and osteogenic differentiation and/or synthesis of ECM [103].

The recruitment and migration of MSCs play significant roles in articular cartilage repair. Once articular cartilage lesions occur, intra- and periarticular MSCs become the primary forces of endogenous cartilage repair. Therefore, there is increasing interest in the endogenous repair strategy for articular cartilage lesions based on MSC recruitment and migration. Chondrocytes that are derived from osteoarthritis patients have perhaps lost the capacity for synthesizing cartilage matrix [104]. Surprisingly, when cultured in 3D cultures or established differentiation conditions, MSCs can gain chondrogenic ability [105]. Various components of the cartilaginous matrix will be formed during the process of chondrogenic differentiation, including glycosaminoglycan, aggrecan and collagen type II [106]. The reconstructed ECM plays an important role in establishing relations with MSCs as well as chondrocytes and regulating cell proliferation, differentiation and migration $[107,108]$. In vivo, a co-culture system 
of chondrocytes and MSCs is formed when MSCs migrate to injured cartilage. On the one hand, in this system chondrocytes can mediate MSC chondrogenic differentiation [109,110]. On the other hand, MSCs can enhance chondrocyte regenerative ability [111,112]. In addition, previous studies found that chondrocytes could increase matrix production and the capacity for chondrogenic differentiation of adipose tissue-derived MSCs $[109,113,114]$ as well as BM-MSCs $[109,110]$. Chondrogenic genes, such as COL2A1 and $A C N A$, are also upregulated when co-cultured with chondrocytes and BM-MSCs [111,113]. The expression of hypertrophic and fibrotic genes is also reduced in osteoarthritis-derived chondrocytes when interacting with MSCs [109,111,114]. Moreover, chondrocytes co-cultured with BM-MSCs could increase hyaluronan synthesis [115], which is the protective agent of articular cartilage [116].

Recently, FGF2 has been shown to play an important role in the process of cartilage regeneration. FGF2 is an endogenous and inherent growth factor in articular cartilage [117,118], and it was first discovered in the ECM of chondrocytes and osteoblasts [117]. It can bind to heparan sulfate proteoglycan and is stored in the ECM of articular cartilage $[117,118]$. When cartilage damage occurs or cartilage degenerates, FGF2 is quickly released from the ECM and activates the ERK signaling pathway $[58,106]$. As the main stimulus of ERK, FGF2 sustains activation of ERK. After the ERK signaling pathway is activated, a series of responses occur. The most important biological function of FGF2 relating to cartilage regeneration is that it increases the expression of the chondrocyte gene after cartilage damage or degeneration. Thus, FGF2 can promote cartilage regeneration by activating the ERK signaling pathway. However, the effect of FGF2 on cartilage has two sides: it can promote the regeneration and repair of cartilage, but it can also promote cartilage degradation. Its biological function is mainly related to the receptors it binds. The FGFRs expressed in adult articular chondrocytes have four forms, FGFR1-FGFR4, and FGFR1 and FGFR3 are the major types [119]. When FGF2 binds to FGFR3, it can promote the repair of articular cartilage. However, when FGF2 binds to FGFR1, it can promote the degeneration of articular cartilage.

When FGF2 binds to FGFR1, the progress of phosphorylation of the receptor occurs [38], which in turn activates two key signaling mediators: Ras and PKC $\delta$ [119]. These molecules then integrate their signal inputs into the Raf-MEK1/2-ERK1/2 cascade to regulate the targeted gene's expression [120]. After the activation of two key transcription factors (ELK1 and RUNX2), the expression of a range of genes, including MMP13, ADAMTS4, ADAMTS5, COL1OA1 and VEGF, is upregulated, ultimately promoting cartilage degradation. The p38 and JNK pathways are also activated by PKC $\delta$ [120], and the effect also promotes ECM degeneration and inhibits anabolic activity [120]. Yan et al. proposed that FGF2 triggered the consumption of proteoglycans in cartilage and found that FGF2 could inhibit the long-term accumulation of proteoglycans in articular chondrocytes in in vitro and in vivo studies [119].

By contrast, when FGF2 binds to FGFR3, it can promote the repair of articular cartilage. It regulates proliferation, differentiation and matrix production of articular chondrocytes and can promote the synthesis of proteoglycan. FGFR3 plays a crucial anabolic role after being activated by FGF2 and promotes the repair of articular cartilage. Therefore, it is possible to prevent cartilage degradation and promote cartilage repair by researching FGFR1 antagonists and FGFR3-activating agonists. Species differences regarding the effect of FGF2 may be mainly related to the corresponding receptor differences. Human articular cartilage mainly contains FGFR1 and FGFR3, whereas the articular cartilage of mice mainly contains FGFR2 and FGFR4. However, the reasons for these differences require further research [121].

In recent years, more and more studies have investigated the repair of cartilage damage by FGF2 [44-46]. FGF2 has also been proven to promote the repair of full-thickness cartilage damage [45,122], but its concrete mechanism is still unclear. In addition to activating the FGF/FGFR2 pathway, FGF2 also activates the P13K-Akt pathway to promote the proliferation of BM-MSCs and promote their differentiation into cartilage by regulating the expression of SOX9, a key transcription factor of cartilage. Zhang found that the cartilage layer and chondrocytes are not effective targets of FGF2 [ZHANG Z. FUNDAMENTAL RESEARCH ON OPTIMAL DELIVERY OF FGF2 TO PROMOTE ARTICULAR CARTILAGE REPAIR (2OI5), UNPUBLISHED DATA]. If FGF2 acts directly on the cartilage layer or chondrocytes, it can accelerate the degradation of the ECM of the cartilage. However, when FGF2 acts on subchondral bone, it can promote cartilage formation. Zhang also found that if FGF2 acts directly on the cartilage layer, it can accelerate the degradation of the ECM of the cartilage and inhibit the expression of endogenous growth factors related to cartilage repair, which indicates that the cartilage layer is not the site where FGF2 plays its part. However, the study surprisingly found that FGF2 can effectively regulate subchondral bone to promote cartilage formation, mainly by upregulating the expression of endogenous growth factors such as BMP2 and BMP4 in the bone. Other studies have demonstrated that FGF2 can regulate the expression of SOX9 and promote the formation of cartilage by inducing proliferation and differentiation of BM-MSCs into chondrocytes. Li et al. found that FGF2 promoted cartilage repair by 
Table 1. The application of FGF2 in tendon-to-bone healing and cartilage repair.

\begin{tabular}{|c|c|c|c|c|}
\hline Model & Cells & Technique & Application & Ref. \\
\hline Rabbit & - & In vivo & Tendon-to-bone healing & [65] \\
\hline- & Human BM-MSCs & In vitro & Tendon-to-bone healing & [66] \\
\hline Rabbit & Rabbit BM-MSCs & In vitro and in vivo & Tendon-to-bone healing & [67] \\
\hline Pig & - & In vitro & Tendon-to-bone healing & [68] \\
\hline- & Human fibroblasts & In vitro & Tendon-to-bone healing & [95] \\
\hline- & Rat tenocytes & In vitro & Tendon-to-bone healing & [77] \\
\hline Chicken & HEK293 cells & In vitro and in vivo & Tendon-to-bone healing & [96] \\
\hline Chicken & Chicken tenocytes & In vitro and in vivo & Tendon-to-bone healing & [97] \\
\hline Rat & Rat periosteum-derived cells & In vitro and in vivo & Tendon-to-bone healing & [98] \\
\hline Rabbit & - & In vitro and in vivo & Tendon-to-bone healing & [102] \\
\hline- & Pig chondrocytes & In vitro & Cartilage repair & [118] \\
\hline- & Human chondrocytes & In vitro & Cartilage repair & [119] \\
\hline- & Human BM-MSCs & In vitro & Cartilage repair & [122] \\
\hline Rabbit & Rabbit BM-MSCs & In vitro and in vivo & Cartilage repair & [3] \\
\hline Rabbit & - & In vitro and in vivo & Cartilage repair & [123] \\
\hline Mouse & Human periosteal cells & In vitro and in vivo & Cartilage repair & [124] \\
\hline Murine & - & In vitro and in vivo & Cartilage repair & [125] \\
\hline
\end{tabular}

upregulating multiple growth factors, including TGF- $\beta 2$, VEGF, FGF2, BMP2, BMP3 and BMP4, which play key roles in the repair of cartilage [123]. In addition, Mendes et al. explored the effect of multiple growth factors on chondrogenic differentiation of human periosteum-derived MSCs in vitro and found that $100 \mathrm{ng} / \mathrm{ml}$ of BMP6 and BMP2 and low concentrations of FGF2 could increase the expression of SOX9 and COL2A1 as well as the matrix molecule aggrecan compared with control groups [124]. Therefore, low concentrations of FGF2 might be conducive to chondrogenic differentiation and chondrogenic repair. In addition, FGF2 has many isoforms, and Burt et al. found that overexpression of the high molecular weight isoforms of FGF2 in bone results in catabolic activity in joint cartilage and bone and finally leads to the occurrence of osteoarthropathy (Table 1) [125].

\section{Conclusion}

As a mitotic promoter and key factor regulating the process of tendon-to-bone healing and cartilage repair, FGF2 has the potential to accelerate tendon-to-bone healing and cartilage repair. It may provide a new direction and therapy for tendon-to-bone healing and cartilage repair in future clinical practice.

In terms of mechanism, FGF2 participates in accelerating tendon-to-bone healing and cartilage repair primarily by binding its specific receptors. As for tendon-to-bone healing, FGF2 not only promotes the revascularization of grafts but also contributes to the formation of new bone - two main factors that determine the tendon-to-bone healing process. With regard to cartilage repair, FGF2 principally recruits MSCs around the injured area and acts directly on subchondral bone to facilitate cartilage repair. There may be more complicated and detailed FGF2 mechanisms relevant to tendon-to-bone healing and cartilage repair that require further efforts to explore and illuminate.

\section{Future perspective}

Tendon-to-bone healing and cartilage repair are major problems worldwide, plaguing the majority of clinicians. Although traditional treatments and emerging therapies relieve pain for a short period of time and improve motor function to some degree, long-term results are still not satisfactory for patients. Thus far, there is still no best therapy for tendon-to-bone healing and cartilage repair. The key to tendon-to-bone healing is the revascularization of grafts and the formation of new bone. Therefore, any treatments should be based on this. The core of cartilage repair is to quickly form an ECM with good viscoelasticity and mechanical strength. In addition, the ECM should be well integrated and compatible with normal cartilage tissue.

As a key factor regulating the process of tendon-to-bone healing and cartilage repair, FGF2 has attracted the attention of many scholars and has achieved many good results. Further studies should focus on further illustrating 
the mechanism of the effect of FGF2 on tendon-to-bone healing and cartilage repair as well as more animal experiments and preclinical studies so as to lay a solid foundation for future clinical application.

\section{Executive summary}

- In this article, the authors reviewed the effect of FGF2 on the promotion of tendon-to-bone healing and cartilage repair.

FGF family members

- At present, 22 FGF-encoding genes have been identified in the human genome, and they can interact with specific receptors on cell membranes to mediate various biological and pathophysiological processes, including embryonic development, angiogenesis, mitogenesis, metabolism regulation, cellular differentiation, cell migration and tissue injury repair.

FGFRs

- Thus far, four FGFRs have been discovered. FGFRs are typical transmembrane tyrosine kinase receptors that are expressed in most tissue cells and mediate the transmission of FGFs into the cytoplasm.

The discovery \& application of FGF2

- The human FGF2 gene is a single-copy gene located on the short arm of chromosome 4, with a total length greater than $40 \mathrm{~kb}$. It plays an important role in tendon-to-bone healing, cartilage repair, bone repair and nerve regeneration.

The effect of FGF2 on tendon-to-bone healing

- In this section, the authors discussed the application of an FGF2 solution as well as gene transfer of FGF2 to promote tendon-to-bone healing. FGF2 can accelerate revascularization of ligaments and promote the formation of new bone - two key factors contributing to tendon-to-bone healing. Therefore, FGF2 plays a key role in promoting tendon-to-bone healing.

The effect of FGF2 on cartilage repair

- On the one hand, FGF2 can promote the recruitment and migration of mesenchymal stem cells, which play significant roles in cartilage repair. On the other hand, FGF2 promotes cartilage repair by acting on subchondral bone.

Conclusion

- FGF2 has positive effects on the promotion of tendon-to-bone healing and cartilage repair.

\section{Author contributions}

Y Li conceived, drafted and finalized the manuscript. J Zhang contributed to writing and finalizing the manuscript. All authors read and approved the final manuscript.

Financial \& competing interests disclosure

This work was financially supported by the Guizhou Science and Technology Department (Gui Zou; grant number LH [2017]7105) and Qian Wei Ji Ban Han (grant number 2017-24). The authors have no other relevant affiliations or financial involvement with any organization or entity with a financial interest in or financial conflict with the subject matter or materials discussed in the manuscript apart from those disclosed.

No writing assistance was utilized in the production of this manuscript.

\section{Open access}

This work is licensed under the Attribution-NonCommercial-NoDerivatives 4.0 Unported License. To view a copy of this license, visit http://creativecommons.org/licenses/by-nc-nd/4.0/

\section{References}

1. Asahara H, Inui M, Lotz MK. Tendons and ligaments: connecting developmental biology to musculoskeletal disease pathogenesis. J. Bone Miner. Res. 32(9), 1773-1782 (2017).

2. Beynnon BD, Johnson RJ, Abate JA, Fleming BC, Nichols CE. Treatment of anterior cruciate ligament injuries, part 2. Am. J. Sports Med. 33(11), 1751-1767 (2005).

3. Baykan MA. Current concepts in ACL reconstruction. J. Sports Sci. Med. 7(3), 424-431 (2008).

4. Moosmayer S, Smith HJ, Tariq R, Larmo A. Prevalence and characteristics of asymptomatic tears of the rotator cuff: an ultrasonographic and clinical study. J. Bone Joint Surg. Br. 91(2), 196-200 (2009).

5. Gerber C, Schneeberger AG, Perren SM, Nyffeler RW. Experimental rotator cuff repair. A preliminary study. J. Bone Joint Surg. Am. 81(9), 1281-1290 (1999). 
6. Chen CH. Graft healing in anterior cruciate ligament reconstruction. BMC Sports Sci. Med. Rehabil. 1(1), 21 (2009).

7. Arendt EA. Anterior cruciate ligament injuries. Curr. Womens Health Rep. 1(3), 211-217 (2001).

8. Benjamin M, Kumai T, Milz S, Boszczyk BM, Boszczyk AA, Ralphs JR. The skeletal attachment of tendons—tendon "entheses." Comp. Biochem. Physiol. A Mol. Integr. Physiol. 133(4), 931-945 (2002).

9. Benjamin M, Toumi H, Ralphs JR, Bydder G, Best TM, Milz S. Where tendons and ligaments meet bone: attachment sites ('entheses') in relation to exercise and/or mechanical load. J. Anat. 208(4), 471-490 (2006).

10. Thomopoulos S, Genin GM, Galatz LM. The development and morphogenesis of the tendon-to-bone insertion-what development can teach us about healing. J. Musculoskelet. Neuronal Interact. 10(1), 35-45 (2010).

11. Thomopoulos S, Williams GR, Gimbel JA, Favata M, Soslowsky LJ. Variation of biomechanical, structural, and compositional properties along the tendon to bone insertion site. J. Orthop. Res. 21(3), 413-419 (2003).

12. Moffat KL, Sun WH, Pena PE et al. Characterization of the structure-function relationship at the ligament-to-bone interface. Proc. Natl Acad. Sci. U. S. A. 105(23), 7947-7952 (2008).

13. Wong MW, Qin L, Tai JK, Lee SK, Leung KS, Chan KM. Engineered allogeneic chondrocyte pellet for reconstruction of fibrocartilage zone at bone-tendon junction-a preliminary histological observation. J. Biomed. Mater. Res. B Appl. Biomater. 70(2), 362-367 (2004).

14. Gulotta LV, Kovacevic D, Ying L, Ehteshami JR, Montgomery S, Rodeo SA. Augmentation of tendon-to-bone healing with a magnesium-based bone adhesive. Am. J. Sports Med. 36(7), 1290-1297 (2008).

15. Rodeo SA, Arnoczky SP, Torzilli PA, Hidaka C, Warren RF. Tendon-healing in a bone tunnel. A biomechanical and histological study in the dog. J. Bone Joint Surg. Am. 75(12), 1795-1803 (1993).

16. Tomita F, Yasuda K, Mikami S, Sakai T, Yamazaki S, Tohyama H. Comparisons of intraosseous graft healing between the doubled flexor tendon graft and the bone-patellar tendon-bone graft in anterior cruciate ligament reconstruction. Arthroscopy 17(5), 461-476 (2001).

17. Newsham-West R, Nicholson H, Walton M, Milburn P. Long-term morphology of a healing bone-tendon interface: a histological observation in the sheep model. J. Anat. 210(3), 318-327 (2007).

18. Milz S, Rufai A, Buettner A, Putz R, Ralphs JR, Benjamin M. Three-dimensional reconstructions of the Achilles tendon insertion in man. J. Anat. 200(2), 145-152 (2002).

19. Liu SH, Panossian V, al-Shaikh R et al. Morphology and matrix composition during early tendon to bone healing. Clin. Orthop. Relat. Res. 339(2), 253-260 (1997).

20. Woo SL-Y, Buckwalter JA. Injury and repair of the musculoskeletal soft tissues. Am. Acad. Orthop. Surg. 6(6) 133-166 (1988).

21. Rothrauff BB, Tuan RS. Cellular therapy in bone-tendon interface regeneration. Organogenesis 10(1), 13-28 (2014).

22. Cooper RR, Misol S. Tendon and ligament insertion. A light and electron microscopic study. J. Bone Joint Surg. Am. 52(1), 1-20 (1970).

23. Yun YR, Won JE, Jeon E et al. Fibroblast growth factors: biology, function, and application for tissue regeneration. J. Tissue Eng. 2010(7), 1-18 (2010).

24. Kontturi LS, Järvinen E, Muhonen V et al. An injectable, in situ forming type II collagen/hyaluronic acid hydrogel vehicle for chondrocyte delivery in cartilage tissue engineering. Drug Deliv. Transl. Res. 4(2), 149-158 (2014).

25. Vinatier C, Mrugala D, Jorgensen C, Guicheux J, Noël D. Cartilage engineering: a crucial combination of cells, biomaterials and biofactors. Trends Biotechnol. 27(5), 307-314 (2009).

26. Temenoff JS, Mikos AG. Review: tissue engineering for regeneration of articular cartilage. Biomaterials 21(5), 431-440 (2000).

27. Huey DJ, Hu JC, Athanasiou KA. Unlike bone, cartilage regeneration remains elusive. Science 338(6109), 917-921 (2012).

28. Nukavarapu SP, Dorcemus DL. Osteochondral tissue engineering: current strategies and challenges. Biotechnol. Adv. 31(5), 706-721 (2013).

29. Beris AE, Lykissas MG, Papageorgiou CD, Georgoulis AD. Advances in articular cartilage repair. Injury 36(Suppl. 4), S14-S23 (2005).

30. Lotz MK, Caramés B. Autophagy and cartilage homeostasis mechanisms in joint health, aging and OA. Nat. Rev. Rheumatol. 7(10), 579-587 (2011).

31. Gavenis K, Schmidt-Rohlfing B, Andereya S, Mumme T, Schneider U, Mueller-Rath R. A cell-free collagen type I device for the treatment of focal cartilage defects. Artif. Organs 34(1), 79-83 (2010).

32. Kon E, Delcogliano M, Filardo G et al. A novel nano-composite multi-layered biomaterial for treatment of osteochondral lesions: technique note and an early stability pilot clinical trial. Injury 41(7), 693-701 (2010).

33. Coburn JM, Gibson M, Monagle S, Patterson Z, Elisseeff JH. Bioinspired nanofibers support chondrogenesis for articular cartilage repair. Proc. Natl Acad. Sci. U. S. A. 109(25), 10012-10017 (2012).

34. Smith GD, Knutsen G, Richardson JB. A clinical review of cartilage repair techniques. J. Bone Joint Surg. Br. 87(4), 445-449 (2005).

35. Hunziker EB, Lippuner K, Keel MJB et al. An educational review of cartilage repair: precepts \& practice-myths \& misconceptions-progress \& prospects. Osteoarthritis Cartilage 23(3), 334-350 (2015). 
36. Niethammer TR, Pietschmann MF, Horng A et al. Graft hypertrophy of matrix-based autologous chondrocyte implantation: a two-year follow-up study of NOVOCART 3D implantation in the knee. Knee Surg. Sports Traumatol. Arthrosc. 22(6), 1329-1336 (2014).

37. Ao Y, Li Z, You Q et al. The use of particulated juvenile allograft cartilage for the repair of porcine articular cartilage defects. Am. J. Sports Med. 47(10), 2308-2315 (2019).

38. Katagiri H, Mendes LF, Luyten FP. Definition of a critical size osteochondral knee defect and its negative effect on the surrounding articular cartilage in the rat. Osteoarthritis Cartilage 25(9), 1531-1540 (2017).

39. Filardo G, Kon E, Di Martino A et al. Treatment of knee osteochondritis dissecans with a cell-free biomimetic osteochondral scaffold: clinical and imaging evaluation at 2-year follow-up. Am. J. Sports Med. 41(8), 1786-1793 (2013).

40. Vanlauwe J, Saris DBF, Victor J et al. Five-year outcome of characterized chondrocyte implantation versus microfracture for symptomatic cartilage defects of the knee: early treatment matters. Am. J. Sports Med. 39(12), 2566-2574 (2011).

41. Bentley G, Biant LC, Vijayan S, Macmull S, Skinner JA, Carrington RW. Minimum ten-year results of a prospective randomized study of autologous chondrocyte implantation versus mosaicplasty for symptomatic articular cartilage lesions of the knee. J. Bone Joint Surg. Br. 94(4), 504-509 (2012).

42. Powers CJ, McLeskey SW, Wellstein A. Fibroblast growth factors, their receptors and signaling. Endocr. Relat. Cancer 7(3), 165-197 (2000).

43. Ide J, Kikukawa K, Hirose $\mathrm{J}$ et al. The effect of a local application of fibroblast growth factor- 2 on tendon-to-bone remodeling in rats with acute injury and repair of the supraspinatus tendon. J. Shoulder Elbow Surg. 18(3), 391-398 (2009).

44. Yamamoto T, Wakitani S, Imoto $\mathrm{K}$ et al. Fibroblast growth factor-2 promotes the repair of partial thickness defects of articular cartilage in immature rabbits but not in mature rabbits. Osteoarthritis Cartilage 12(8), 636-641 (2004).

45. Chuma H, Mizuta H, Kudo S, Takagi K, Hiraki Y. One day exposure to FGF-2 was sufficient for the regenerative repair of full-thickness defects of articular cartilage in rabbits. Osteoarthritis Cartilage 12(10), 834-842 (2004).

46. Li X, Su G, Wang J et al. Exogenous bFGF promotes articular cartilage repair via up-regulation of multiple growth factors. Osteoarthritis Cartilage 21(10), 1567-1575 (2013).

47. Wang H, Zou Q, Boerman OC et al. Combined delivery of BMP-2 and bFGF from nanostructured colloidal gelatin gels and its effect on bone regeneration in vivo. J. Control. Release 166(2), 172-181 (2013).

48. Perrimon N, Bernfield M. Specificities of heparan sulphate proteoglycans in developmental processes. Nature 404(6779), 725-728 (2000).

49. Itoh N, Ornitz DM. Evolution of the Fgf and Fgfr gene families. Trends Genet. 20(11), 563-569 (2004).

50. Mohammadi M, Olsen SK, Ibrahimi OA. Structural basis for fibroblast growth factor receptor activation. Cytokine Growth Factor Rev. 16(2), 107-137 (2005).

51. Burgess WH, Friesel R, Winkles JA. Structure-function studies of FGF-1: dissociation and partial reconstitution of certain of its biological activities. Molecular reproduction and development. Mol. Reprod. Dev. 39(1), 56-61 (1994).

52. Rizzino A, Rosfjord E. Transcriptional regulation of the murine k-fgf gene. Mol. Reprod. Dev. 39(1), 106-111 (1994).

53. Jaye M, Howk R, Burgess W, Ricca GA. Human endothelial cell growth factor: cloning, nucleotide sequence, and chromosome localization. Science 233(4763), 541-545 (1986).

54. Asada M, Shinomiya M, Suzuki M et al. Glycosaminoglycan affinity of the complete fibroblast growth factor family. Biochim. Biophys. Acta 1790(1), 40-48 (2008).

55. Schlessinger J, Plotnikov AN, Ibrahimi OA et al. Crystal structure of a ternary FGF-FGFR-heparin complex reveals a dual role for heparin in FGFR binding and dimerization. Mol. Cell 6(3), 743-750 (2000).

56. Furdui CM, Lew ED, Schlessinger J, Anderson KS. Autophosphorylation of FGFR1 kinase is mediated by a sequential and precisely ordered reaction. Mol. Cell 21(5), 711-717 (2006).

57. Cool SM, Snyman CP, Nurcombe V et al. Temporal expression of fibroblast growth factor receptors during primary ligament repair. Knee Surg. Sports Traumatol. Arthrosc. 12(5), 490-496 (2004).

58. Yan D, Chen D, Cool SM et al. Fibroblast growth factor receptor 1 is principally responsible for fibroblast growth factor 2-induced catabolic activities in human articular chondrocytes. Arthritis Res. Ther. 13(4), R130 (2011).

59. Yuanyuan L, Yan J, Yanfang H et al. Clinical features and FGFR3 gene mutation of patients with achondroplasia. Chin. J. Osteoporosis Bone Mineral Salt Dis. 000(003), 210-214 (2015).

60. Brancaleoni GH, Lourenzoni MR, Degrève L. Study of the influence of ethanol on basic fibroblast growth factor structure. Genet. Mol. Res. 5(2), 350-372 (2006).

61. Yao ZZ, Hu AX, Liu XS. DUSP19 regulates IL-1 $\beta$-induced apoptosis and MMPs expression in rat chondrocytes through JAK2/STAT3 signaling pathway. Biomed. Pharmacother. 96(1), 1209-1215 (2017).

62. Park JS, Lee J, Lim MA et al. JAK2-STAT3 blockade by AG490 suppresses autoimmune arthritis in mice via reciprocal regulation of regulatory T cells and Th17 cells. J. Immunol. 192(9), 4417-4424 (2014). 
63. Ralles S, Agel J, Obermeier M, Tompkins M. Incidence of secondary intra-articular injuries with time to anterior cruciate ligament reconstruction. Am. J. Sports Med. 43(6), 1373-1379 (2015).

64. Chen ZD, Cai YY, Wu J, Lin B, Ye YH. Study on differentiation of sputum cells from bone marrow mesenchymal stem cells induced by fibroblast growth factor. Zhongguo Guyuguanjie Sunshang Zazhi 32(8), 819-822 (2017).

65. Kobayashi M, Itoi E, Minagawa $\mathrm{H}$ et al. Expression of growth factors in the early phase of supraspinatus tendon healing in rabbits. $J$. Shoulder Elbow Surg. 15(3), 1-7 (2006).

66. Hankemeier S, Keus M, Zeichen J et al. Modulation of proliferation and differentiation of human bone marrow stromal cells by fibroblast growth factor 2: potential implications for tissue engineering of tendons and ligaments. Tissue Eng. 11(1-2), 41-49 (2005).

67. Zhang C, Li Q, Deng $S$ et al. bFGF- and CaPP-loaded fibrin clots enhance the bioactivity of the tendon-bone interface to augment healing. Am. J. Sports Med. 44(8), 1972-1982 (2016).

68. MacLean SB, Gratzer PF. Effect of basic fibroblast growth factor on the cellular repopulation of decellularized anterior cruciate ligament allografts. J. Tissue Eng. Regen. Med. 5(3), 201-209 (2011).

69. Hildebrand KA, Frank CB, Hart DA. Gene intervention in ligament and tendon: current status, challenges, future directions. Gene Ther. 11(4), 368-378 (2004).

70. Huard J, Li Y, Peng H, Fu FH. Gene therapy and tissue engineering for sports medicine. J. Gene Med. 5(2), 93-108 (2003).

71. Nixon AJ, Watts AE, Schnabel LV. Cell- and gene-based approaches to tendon regeneration. J. Shoulder Elbow Surg. 21(2), 278-294 (2012).

72. Woo SL, Jia F, Zou L, Gabriel MT. Functional tissue engineering for ligament healing: potential of antisense gene therapy. Ann. Biomed. Eng. 32(3), 342-351 (2004).

73. Attia E, Brown H, Henshaw R, George S, Hannafin JA. Patterns of gene expression in a rabbit partial anterior cruciate ligament transection model: the potential role of mechanical forces. Am. J. Sports Med. 38(2), 348-356 (2010).

74. Wang Y, Tang Z, Xue R et al. TGF- $\beta 1$ promoted MMP-2 mediated wound healing of anterior cruciate ligament fibroblasts through NF-кB. Connect. Tissue Res. 52(3), 218-225 (2011).

75. Basile P, Dadali T, Jacobson J et al. Freeze-dried tendon allografts as tissue-engineering scaffolds for Gdf5 gene delivery. Mol. Ther. 16(3), 466-473 (2008).

76. Wang XT, Liu PY, Tang JB et al. Tendon healing in vitro: adeno-associated virus-2 effectively transduces intrasynovial tenocytes with persistent expression of the transgene, but other serotypes do not. Plast. Reconstr. Surg. 119(1), 227-334 (2007).

77. Wang XT, Liu PY, Xin KQ, Tang JB. Tendon healing in vitro: bFGF gene transfer to tenocytes by adeno-associated viral vectors promotes expression of collagen genes. J. Hand Surg. Am. 30(6), 1255-1261 (2005).

78. Nakamura N, Shino K, Natsuume T et al. Early biological effect of in vivo gene transfer of platelet-derived growth factor (PDGF)-B into healing patellar ligament. Gene Ther. 5(9), 1165-1170 (1998).

79. Suwalski A, Dabboue H, Delalande A et al. Accelerated Achilles tendon healing by PDGF gene delivery with mesoporous silica nanoparticles. Biomaterials 31(19), 5237-5245 (2010).

80. Uggen JC, Dines J, Uggen CW et al. Tendon gene therapy modulates the local repair environment in the shoulder. J. Am. Osteopath. Assoc. 105(1), 20-21 (2005).

81. Wang XT, Liu PY, Tang JB. Tendon healing in vitro: genetic modification of tenocytes with exogenous PDGF gene and promotion of collagen gene expression. J. Hand Surg. Am. 29(5), 884-890 (2004).

82. Wang XT, Liu PY, Tang JB. Tendon healing in vitro: modification of tenocytes with exogenous vascular endothelial growth factor gene increases expression of transforming growth factor beta but minimally affects expression of collagen genes. J. Hand Surg. Am. 30(2), 222-229 (2005).

83. Bolt $\mathrm{P}$, Clerk AN, Luu HH et al. BMP-14 gene therapy increases tendon tensile strength in a rat model of Achilles tendon injury. J. Bone Joint Surg. Am. 89(6), 1315-1320 (2007).

84. Lou J, Tu Y, Burns M, Silva MJ, Manske P. BMP-12 gene transfer augmentation of lacerated tendon repair. J. Orthop. Res. 19(6), 1199-1202 (2001).

85. Ma Y, Zhang X, Wang J et al. Effect of bone morphogenetic protein-12 gene transfer on posterior cruciate ligament healing in a rabbit model. Am. J. Sports Med. 37(3), 599-609 (2009).

86. Majewski M, Betz O, Ochsner PE, Liu F, Porter RM, Evans CH. Ex vivo adenoviral transfer of bone morphogenetic protein 12 (BMP-12) cDNA improves Achilles tendon healing in a rat model. Gene Ther. 15(16), 1139-1146 (2008).

87. Mehta V, Kang Q, Luo J, He TC, Haydon RC, Mass DP. Characterization of adenovirus-mediated gene transfer in rabbit flexor tendons. J. Hand Surg. Am. 30(1), 136-141 (2005).

88. Rickert M, Wang H, Wieloch P et al. Adenovirus-mediated gene transfer of growth and differentiation factor-5 into tenocytes and the healing rat Achilles tendon. Connect. Tissue Res. 46(4-5), 175-183 (2005). 
89. Schnabel LV, Lynch ME, van der Meulen MC, Yeager AE, Kornatowski MA, Nixon AJ. Mesenchymal stem cells and insulin-like growth factor-I gene-enhanced mesenchymal stem cells improve structural aspects of healing in equine flexor digitorum superficialis tendons. $J$. Orthop. Res. 27(10), 1392-1398 (2009).

90. Steinert AF, Weber M, Kunz M et al. In situ IGF-1 gene delivery to cells emerging from the injured anterior cruciate ligament. Biomaterials 29(7), 904-916 (2008).

91. Hou Y, Mao Z, Wei X et al. The roles of TGF-beta1 gene transfer on collagen formation during Achilles tendon healing. Biochem. Biophys. Res. Commun. 383(2), 235-239 (2009).

92. Majewski M, Porter RM, Betz OB et al. Improvement of tendon repair using muscle grafts transduced with TGF- $\beta 1$ cDNA. Eur. Cell. Mater. 23, 94-101 (2012).

93. Wei X, Mao Z, Hou Y et al. Local administration of TGFß-1/VEGF165 gene-transduced bone mesenchymal stem cells for Achilles allograft replacement of the anterior cruciate ligament in rabbits. Biochem. Biophys. Res. Commun. 406(2), 204-210 (2011).

94. Gulotta LV, Kovacevic D, Packer JD, Deng XH, Rodeo SA. Bone marrow-derived mesenchymal stem cells transduced with scleraxis improve rotator cuff healing in a rat model. Am. J. Sports Med. 39(6), 1282-1289 (2011).

95. Madry H, Kohn D, Cucchiarini M. Direct FGF-2 gene transfer via recombinant adeno-associated virus vectors stimulates cell proliferation, collagen production, and the repair of experimental lesions in the human ACL. Sports Med. 41(1), 194-202 (2013).

96. Tang JB, Cao Y, Zhu B, Xin KQ, Wang XT, Liu PY. Adeno-associated virus-2-mediated bFGF gene transfer to digital flexor tendons significantly increases healing strength. An in vivo study. J. Bone Joint Surg. Am. 90(5), 1078-1089 (2008).

97. Yang QQ, Shao YX, Zhang LZ, Zhou YL. Therapeutic strategies for flexor tendon healing by nanoparticle-mediated co-delivery of bFGF and VEGFA genes. Colloids Surf. B Biointerfaces 164, 165-176 (2018).

98. Yin J, Qiu S, Shi B et al. Controlled release of FGF-2 and BMP-2 in tissue engineered periosteum promotes bone repair in rats. Biomed. Mater. 13(2), 1-10 (2018).

99. Ikpegbu E, Basta L, Clements DN et al. FGF-2 promotes osteocyte differentiation through increased E11/podoplanin expression. J. Cell Physiol. 233(7), 5334-5347 (2018).

100. Jacob A, Smith C, Partanen J et al. Fibroblast growth factor receptor 1 signaling in the osteo-chondrogenic cell lineage regulates sequential steps of osteoblast maturation. Dev. Biol. 296(2), 315-328 (2006).

101. Raman M, Chen W, Cobb MH. Differential regulation and properties of MAPKs. Oncogene 26(22), 3100-3112 (2007).

102. Lee SH, Park YB, Moon HS et al. The role of rhFGF-2 soaked polymer membrane for enhancement of guided bone regeneration. J. Biomater. Sci. Polym. Ed. 29(7-9), 825-843 (2018).

103. Santo VE, Gomes ME, Mano JF, Reis RL. Controlled release strategies for bone, cartilage, and osteochondral engineering-Part I: recapitulation of native tissue healing and variables for the design of delivery systems. Tissue Eng. Part B Rev. 19(4), 308-326 (2013).

104. Gao ZQ, Guo X, Duan C, Ma W et al. Altered aggrecan synthesis and collagen expression profiles in chondrocytes from patients with Kashin-Beck disease and osteoarthritis. J. Int. Med. Res. 40(4), 1325-1334 (2012).

105. Lai JH, Rogan H, Kajiyama G et al. Interaction between osteoarthritic chondrocytes and adipose-derived stem cells is dependent on cell distribution in three-dimension and transforming growth factor-beta3 induction. Tissue Eng. Part A 21(5-6), 992-1002 (2015).

106. Meretoja VV, Dahlin RL, Kasper FK, Mikos AG. Enhanced chondrogenesis in co-cultures with articular chondrocytes and mesenchymal stem cells. Biomaterials 33(27), 6362-6369 (2012).

107. Zeltz C, Gullberg D. The integrin-collagen connection—a glue for tissue repair? J. Cell Sci. 129(4), 653-664 (2016).

108. Loeser RF. Integrins and chondrocyte-matrix interactions in articular cartilage. Matrix Biol. 39(1), 3911-3916 (2014).

109. Acharya C, Adesida A, Zajac P et al. Enhanced chondrocyte proliferation and mesenchymal stromal cells chondrogenesis in coculture pellets mediate improved cartilage formation. J. Cell. Phys. 227(1), 88-97 (2012).

110. Windt TS, Saris DBF, Slaper-Cortenbach ICM et al. Direct cell-cell contact with chondrocytes is a key mechanism in multipotent mesenchymal stromal cell-mediated chondrogenesis. Tissue Eng. Part A 21(19-20), 2536-2547 (2015).

111. Zhang Q, Chen Y, Wang Q et al. Effect of bone marrow-derived stem cells on chondrocytes from patients with osteoarthritis. Mol. Med. Rep. 13(2), 1795-1800 (2016).

112. Xu L, Wu Y, Xiong Z, Zhou Y, Ye Z, Tan WS. Mesenchymal stem cells reshape and provoke proliferation of articular chondrocytes by paracrine secretion. Sci. Rep. 6(2), 1-13 (2016).

113. Pleumeekers MM, Nimeskern L, Koevoet JLM, Karperien M, Stok KS, van Osch GJVM. Trophic effects of adipose-tissue-derived and bone-marrow-derived mesenchymal stem cells enhance cartilage generation by chondrocytes in co-culture. PLoS One 13(2), 1-23 (2018).

114. Ahmed MR, Mehmood A, Bhatti Fu-R, Khan SN, Riazuddin S. Combination of ADMSCs and chondrocytes reduces hypertrophy and improves the functional properties of osteoarthritic cartilage. Osteoarthritis Cartilage 22(11), 1894-1901 (2014).

115. Antonioli E, Piccinato CA, Nader HB, Cohen M, Goldberg AC, Ferretti M. Modulation of hyaluronan synthesis by the interaction between mesenchymal stem cells and osteoarthritic chondrocytes. Stem Cells Int. 2015, 640218 (2015). 
116. Ishijima M, Nakamura T, Shimizu K et al. Intra-articular hyaluronic acid injection versus oral non-steroidal anti-inflammatory drug for the treatment of knee osteoarthritis: a multi-center, randomized, open-label, non-inferiority trial. Arthritis Res. Ther. 16(1), R18 (2014).

117. Vincent TL, McLean CJ, Full LE, Peston D, Saklatvala J. FGF-2 is bound to perlecan in the pericellular matrix of articular cartilage, where it acts as a chondrocyte mechanotransducer. Osteoarthritis Cartilage 15(7), 752-763 (2007).

118. Vincent T, Hermansson M, Bolton M, Wait R, Saklatvala J. Basic FGF mediates an immediate response of articular cartilage to mechanical injury. Proc. Natl Acad. Sci. U. S. A. 99(12), 8259-8264 (2002).

119. Yan D, Chen D, Im HJ. Fibroblast growth factor-2 promotes catabolism via FGFR1-Ras-Raf-MEK1/2-ERK1/2 axis that coordinates with the PKC $\delta$ pathway in human articular chondrocytes. J. Cell. Biochem. 113(9), 2856-2865 (2012).

120. Im HJ, Muddasani P, Natarajan V et al. Basic fibroblast growth factor stimulates matrix metalloproteinase-13 via the molecular cross-talk between the mitogen-activated protein kinases and protein kinase Cdelta pathways in human adult articular chondrocytes. Biol. Chem. 282(15), 11110-11121 (2007).

121. Li X, Ellman MB, Kroin JS et al. Species-specific biological effects of FGF-2 in articular cartilage: implication for distinct roles within the FGF receptor family. J. Cell. Biochem. 113(7), 2532-2542 (2012).

122. Handorf AM, Li WJ. Fibroblast growth factor-2 primes human mesenchymal stem cells for enhanced chondrogenesis. PLoS One 6(7), 1-11 (2011).

123. Li X, Su G, Wang J et al. Exogenous bFGF promotes articular cartilage repair via up-regulation of multiple growth factors. Osteoarthritis Cartilage 21(10), 1567-1575 (2013).

124. Mendes LF, Tam WL, Chai YC, Geris L, Luyten FP, Roberts SJ. Combinatorial analysis of growth factors reveals the contribution of bone morphogenetic proteins to chondrogenic differentiation of human periosteal cells. Tissue Eng. Part C Methods 22(5), 473-486 (2016).

125. Burt PM, Xiao L, Dealy C et al. FGF2 high molecular weight isoforms contribute to osteoarthropathy in male mice. Endocrinology 157(12), 4602-4614 (2016). 